Please do not remove this page

RMIT

UNIVERSITY

\title{
Prioritizing urban habitats for connectivity conservation: Integrating centrality and ecological metrics
}

Poodat, Fatemeh; Arrowsmith, Colin; Fraser, David; Gordon, Ascelin

https://researchrepository.rmit.edu.au/esploro/outputs/9921862386101341/filesAndLinks?institution=61RMIT_INST\&index=null

Poodat, F., Arrowsmith, C., Fraser, D., \& Gordon, A. (2015). Prioritizing urban habitats for connectivity conservation: Integrating centrality and ecological metrics. Environmental Management, 56, 664-674. https://doi.org/10.1007/s00267-015-0520-2

Document Version: Accepted Manuscript

Published Version: https://doi.org/10.1007/s00267-015-0520-2

Repository homepage: https://researchrepository.rmit.edu.au

(C) 2015 Springer Science+Business Media New York

Downloaded On 2023/04/26 18:24:17 +1000

Please do not remove this page 
Thank you for downloading this document from the RMIT Research Repository.

The RMIT Research Repository is an open access database showcasing the research outputs of RMIT University researchers.

RMIT Research Repository: http://researchbank.rmit.edu.au/

\section{Citation:}

Poodat, F, Arrowsmith, C, Fraser, D and Gordon, A 2015, 'Prioritizing urban habitats for connectivity conservation: Integrating centrality and ecological metrics', Environmental Management, vol. 56, pp. 664-674.

See this record in the RMIT Research Repository at:

https://researchbank.rmit.edu.au/view/rmit:31305

Version: Accepted Manuscript

\section{Copyright Statement:}

(C) 2015 Springer Science+Business Media New York

\section{Link to Published Version:}

http://dx.doi.org/10.1007/s00267-015-0520-2 


\title{
Prioritizing urban habitats for connectivity conservation: integrating centrality and ecological metrics
}

\author{
Fatemeh Poodat, Colin Arrowsmith, David Fraser, Ascelin Gordon
}

\begin{abstract}
Fatemeh Poodat ${ }^{1}$
Affiliation: School of Mathematical and Geospatial Sciences RMIT University Email: f.poodat@gmail.com

Contact details: School of Mathematical and Geospatial Sciences RMIT University

GPO Box 2476 Melbourne Victoria 3001 Australia

+989166123060
\end{abstract}

\section{Colin Arrowsmith}

Affiliation: School of Mathematical and Geospatial Sciences RMIT University

GPO Box 2476 Melbourne Victoria 3001 Australia

Email: colin.arrowsmith@,rmit.edu.au

\section{David Fraser}

Affiliation: School of Mathematical and Geospatial Sciences RMIT University

GPO Box 2476 Melbourne Victoria 3001 Australia

Email: david.fraser@rmit.edu.au

\section{Ascelin Gordon}

Affiliation: School of Global, Urban and Social Studies

GPO Box 2476 Melbourne Victoria 3001 Australia

Email: ascelin.gordon@rmit.edu.au

Note this paper is published as:

Poodat F., Arrowsmith C., Fraser D., Gordon A. (2015) Prioritising urban habitats for connectivity conservation: integrating centrality and metapopulation metrics. Environmental Management. 56: 664-674. doi: 10.1007/s00267-015-0520-2

\footnotetext{
${ }^{1}$ Permanent address: 15/19 Eastern Kanani-Moghadam St., Southern Sarafha St., Saadat Abad, Tehran, Iran
} 


\section{Introduction}

Connectivity between fragmented habitat patches has long been acknowledged as important for biological conservation (Noss et al. 2012; Bennett 2003; Collinge and Forman 1998; Henderson et al. 1985) and ecosystem health (Farina 2000). It is even more important in highly modified landscapes such as urbanised landscapes. These landscapes are often heterogeneous in structure, and fragmentation in habitat patches may result from increased urbanisation. Within and around cities, ecological connectivity between fragments of habitats enhances the dispersal of species and thereby their long-term viability (Wheater 1999; Hamer and McDonnell 2010). Due to the importance of ecological connectivity, the concept has been applied frequently in different studies resulting in a large number of approaches for measuring and quantifying different aspects of connectivity (Kindlmann and Burel 2008). The consequences of choosing which metrics and approaches to use in a given situation is problematic, due to the relative dearth of systematic analyses where the suitability and effectiveness of different measures are compared (Bennett 2003).

Among the approaches for quantifying connectivity, the application of 'graph theory' has shown promise (Urban and Keitt 2001; Galpern et al. 2011; García-Feced et al. 2011). Graph theory is a mathematical theory used for modelling the relationship between individual entities. It consists of finite sets of nodes (or vertices) and links (or lines) (Harary 1969) where the nodes represent the entities and the links represent their relationships. Conservation ecology has utilised graph commonly for the assessment of connectivity, and the fundamentals of the theory are well-presented in several connectivity studies such as Urban and Keitt (2001), Urban et al. (2009), Dale and Fortin (2010) and Galpern et al. (2011).

Large numbers of measures have been developed to address various aspects of connectivity within graph theory (Cantwell and Forman 1993; Urban and Keitt 2001; Pascual-Hortal and Saura 2006; Rothley and Rae 2005; Vasas et al. 2009) and a thorough review of these metrics is provided by Rayfield et al. (2011). Despite this number, few have been comparatively tested with respect to their sensitivity to different components within the network (PascualHortal and Saura 2006; Saura and Pascual-Hortal 2007; Magle et al. 2009; Baranyi et al. 2011). Generally, connectivity metrics provide information on connectivity at either the 'node scale' or the 'network scale'. Network scale metrics provide a single scalar value that 
represents some aspect of connectivity for the network as whole, whereas node scale metrics provide values for each vector linking a node in the network. Node scale metrics allow the contribution of each node to the overall network connectivity to be assessed. Node scale information can also be obtained from network scale metrics by using a "node removal algorithm" (Keitt et al. 1997; Pascual-Hortal and Saura 2006; Urban and Keitt 2001). This technique involves recalculating the network scale metric multiple times with each node in the network removed in turn.

Node scale metrics, such as centrality metrics, have been applied to identify the significance of individual habitat patches in connectivity studies (Bodin and Saura 2010; Bodin and Norberg 2007; Carroll et al. 2012; Estrada and Bodin 2008). Among centrality metrics, $B C$ has been shown to be useful for determining the importance of each node in terms of the traversability of the network (Estrada and Bodin 2008). Patches with a high $B C$ score are more likely to act as important stepping stones for dispersal of a species across the landscape (Baranyi et al. 2011). Degree centrality (DC) is another centrality metric which has the potential to reveal information on the connectivity of a given patch to the adjacent patches at the local scale. Centrality metrics have been shown to be applicable to networks where the links are equally weighted (called an "unweighted network") as well as networks where the links are assigned different weights (a "weighted network”) (Opsahl et al. 2010).

Connectivity metrics can either be labelled as "topological" or "ecological" depending on the types of variables that are incorporated. Topological metrics, which include centrality metrics, originate from the mathematical concept of graph theory and network analysis. These metrics consider the spatial arrangement and configuration of nodes throughout a network. Ecological metrics, however, have grown out of meta-population theory and conservation biology (Rayfield et al. 2011) and specifically address the ecological values associated with habitat networks. Area-based metrics are an example from this group and identify the importance of habitat patches solely based on their structural values such as size, shape and quality (Ferrari et al. 2007; Urban and Keitt 2001).

Identifying habitat patches that play an important role for ecological connectivity in a highly modified landscape is a priority for conservation programs aimed at habitat protection and restoration (Bennett 2003; Lindenmayer and Hobbs 2007; McIntyre and Hobbs 1999). Such knowledge of the connectivity structure of habitats within urban and peri-urban landscapes provides an important basis for advancing conservation efforts. To achieve a realistic 
assessment of connectivity for urban landscapes where particular species can venture into non-habitat areas, responses to moving through the landscape for each species is required (Goodwin, 2003; Kindlmann and Burel, 2008). These responses can be measured in terms of the "cost of moving" from one location to another using ecological measures. The species moving responses to the landscape structure refers to the cost of species movement; species movement characteristics, and habitat preferences which are addressed by ecological metrics. Building weighted networks based on effective distances, distances that incorporate barriers such as roads and rivers, rather than the Euclidean distances, yields more biologically rigorous results in connectivity studies (Magle et al. 2009; Foltête et al. 2012; O'Brien et al. 2006).

In order to incorporate both topological and ecological aspects when ranking habitat patches based on their contribution to connectivity, this study uses another group of metrics called "integrated metrics". Integrated metrics firstly reweight the links in the network using ecological metrics for species dispersal, and then recalculate the topological metrics using this weighted network.

To examine the implication of using integrated metrics, this study analytically compares a selected number of graph-based connectivity metrics according to the way they prioritize habitat patches in an urban context. The eight selected metrics tested are classified into one of the three groups: ecological, topological and integrated metrics and are discussed more fully in section 2.4. All eight metrics were applied to the network of habitat patches for the fattailed dunnart, a threatened mammal species within Greater Melbourne, Australia (Department of Sustainability and Environment 2007).

\section{Material and Methods}

\section{$2.1 \quad$ Study area}

Melbourne is located at the northern end of Port Phillip Bay on the Victorian coastline. The extent of the study area for which connectivity was assessed is shown in Figure 1. The total area is 6320 square kilometres and extends 98.7 kilometres from north to south and 116.5 kilometres east to west. The area incorporates 30 local government municipalities and includes the Urban Growth Boundary established by the Victorian State Government in 2002 (Department of Planning and Community Development 2002). The locations between the Urban Growth Boundary and the outer boundary of the study area are considered as the 
"urban fringe". This area contains important habitat for multiple species threatened by habitat clearing and fragmented from urban development (Hahs and McDonnell 2006).

Melbourne was recognised internationally as the most liveable city in the world in 2011 (The Economist Intelligence Unit 2011). However, the consequences of urbanisation have resulted in a long list of Victoria's flora and fauna species being identified as threatened (Department of Sustainability and Environment 2009a, 2007), many of which are found throughout the study area. With ongoing urbanisation, and the possible impact on habitat, reserves and biodiversity (Gordon et al. 2009; Department of Sustainability and Environment 2009b), it has become necessary to conserve these threatened species through careful management of remanent habitats (Victorian Environmental Assessment Council 2010). Improvement of ecological connectivity is an important component of the activities being undertaken to enhance the condition of flora and fauna.

Fig. 1 Study area. (a) The location of the study area in Melbourne, Australia. (b) The extent of the study area (hatched region) in relation to Greater Melbourne and its Urban Growth Boundary

\section{$2.2 \quad$ Target species}

Connectivity measures for the habitat patch network of the fat-tailed dunnart (Sminthopsis crassicaudata) were applied to the study area. The fat-tailed dunnart is a small nocturnal mammal distributed in central and southern Australia (Figure 2). The species is insectivorous and forages to find invertebrates in open spaces and shelters under logs or rocks in open shrub lands (Frey 1991). The species prefers open grassland habitats (Morton 1976) such as tussock and hummock grassland and rough pasture (Menkhorst and Kenight 2001). These open grasslands are found extensively throughout the northern and western parts of the study area. The fat-tailed dunnart is a mobile terrestrial forager. In some cases distances of up to 400 metres per day have been recorded (Read 1984). Populations of the fat-tailed dunnart still survive in unimproved pasture throughout Victoria (Menkhorst and Bennett 1995). However, the species is listed as 'near threatened' in the advisory list of Victoria (Department of Sustainability and Environment 2007) and is affected by a loss of connectivity in its network. The connectivity of the fat-tailed dunnart's habitat patch network is an important 
factor in the long-term viability of the species is in the study area (Victorian Environmental Assessment Council 2010).

Fig. 2 Fat-tailed dunnart (Sminthopsis crassicaudata); photography by Peter Menkhorst, 2007.

\subsection{Construction of the habitat network for the fat-tailed dunnart}

The habitat map for the fat-tailed dunnart was prepared from data provided for a conservation zoning analysis and has been previously utilised by Gordon et al. (2009). The habitat patches for the species were derived using expert knowledge. This was done via workshop with experts held by the Victorian Government's Department of Environment and Primary Industries to determine the land uses, vegetation and wetland preferences for the dunnart (Gordon et al. 2009). Through a questionnaire experts were asked to define those areas that should be excluded from being habitat for the species. By combining these inputs, a binary raster map of potential habitat/non-habitat for the dunnart was obtained at a 20 metre resolution. As the approach in generating this map was somewhat precautionary, there were a number of instances where pixels classified as suitable habitat were intersected by conflicting land use such as industrial zones, residential zones, or infrastructure such as freeways and highways. These land use types were removed from the derived dunnart habitat raster. From the raster layer, a 'Nearest Neighbour Algorithm' was then applied, to derive individual habitat patches for the dunnart. The habitat patches for the dunnart were then geographically located throughout the North, North West and the West of the study area.

Each habitat patch was reduced to a "node" in the network. The centroid was derived for each patch and used to determine the spatial location of each node. Initially, links were generated to connect one node to the next (see below). These links were non-directional and weighted by the Euclidean distance between patches.

Using a questionnaire followed up with interviews, expert opinions were sought on factors that impede or assist the movement of the dunnart from one location to another. Factors included transport facilities (roads and train lines), native vegetation, hydrology (lakes, rivers 
and dams) and different land use. Slope and elevation throughout the study area were considered negligible. Digital vector maps for each of these input factors were obtained from the Victorian Department of Sustainability and Environment. Each layer was then converted to a raster layer with a 20 metre resolution. Using expert opinion, each raster was then ranked from 1, indicating 'very low resistance' to 100, indicating 'absolute resistance'. This ranking is explained more fully in Beier et al. (2008). Each of the ranked rasters were then combined by taking the maximum value of each pixel over the set of input layers in order to generate a maximum "resistance layer". The resultant resistance layer was a GIS raster in which the resistance values ranged between 1 , referring to the least resistant areas of the landscape, and 100 , referring to the areas most resistant to movement.

The steps involved in the construction of the links are illustrated in Figure 3. In order to develop the links, the Linkage Mapper extension for ArcGIS was used (McRae and Kavanagh 2011). Linkage Mapper was developed to automate mapping of wildlife habitat corridors. As part of its process, the software generates the links for a network of habitat patches. For each link, the software assigns a weighting, called the effective distance, in order to determine the least cost path from moving from one node to another (Adriaensen et al. 2003). The weights were generated by summing each of the cell values that a particular link passes through. Links that were greater than 1000 metres were removed as this distance is considered, from the literature and expert opinion, as the maximum dispersal distance for the dunnart within its lifetime. The software establishing the links for a species network by linking each node to its adjacent nodes (McRae and Kavanagh 2011).

Fig. 3 The steps used for constructing the graph-based connectivity model for the fat-tailed dunnart: (a) the resistance layer (b) the generation of a link network weighted by Euclidean distance (c) the generation of least-cost paths based on the resistance layer and habitat patches for the species and (d) weighting the link by the cost weighted distance of the associated least cost path

Applying Linkage Mapper, the 20 metre cell resolution for determining the least cost path for the entire study area proved to be a computational 'bottleneck'. Therefore the cell size for the final resistance layer was aggregated to 60 metres. A number of smaller habitat zones with less than the 60 metre resolution were eliminated by this process. This was only 0.2 percent 
of the minimum viable size of habitat area of the dunnart and was therefore considered insignificant.

\subsection{Prioritizing habitat patches using connectivity metrics}

Two of the eight metrics compared are topological metrics called $B C$ and $D C$. Two other metrics called area and delta $C$ are considered ecological metrics. The remaining four metrics, called integrated metrics were $B C$ and $D C$, which were each recalculated after the link weights in the network were determined by the probability of dispersal and dispersal flux algorithms.

The significance of each node was determined by applying the eight metrics to the fat-tailed dunnart habitat patch network. Each of the metrics is explained in detail in the following section.

\subsubsection{Topological metrics}

The two centrality metrics selected for comparison with the other metrics were $B C$ and $D C$. These metrics have been shown to provide distinct information on the connectivity contributions of a given node at different scales (Estrada and Bodin 2008). The metrics produce a vector containing the scores of each node in the network. Centrality metrics are based purely on topology and are not associated with the ecological value of a patch and its neighbours. The $D C$ of node $i$ is simply defined as the number of links attached to it (Estrada and Bodin 2008). The $B C$ of a node $k$, is defined as the proportion of shortest paths between each pair of nodes in the network that pass through node $k$ (Freeman 1978):

$$
B C=\sum_{i} \sum_{j} \frac{p(i, k, j)}{p(i, j)} \quad i \neq j \neq k
$$

Here $B C$ stands for Betweenness Centrality, $p(i, j)$ refers to the number of shortest path between node $i$ and $j$ and $p(i, k, j)$ refers to the number of shortest paths between node $i$ and $j$ that pass through node $k$.

Centrality metrics were applied to the network for the fat-tailed dunnart using the thet package within the statistical software $R$ (Opsahl et al. 2010). The tnet package includes a tuning parameter $\alpha$, which determines the extent to which link weights are included in network metric calculations. Setting $\alpha=0$ results in the link weights being ignored while 
values of $\alpha>0$ determine the extent to which the link weights should be included in the calculation. For the $B C$ and $D C$ calculations, $\alpha$ was set to 0 .

\subsubsection{Ecological metrics}

The metrics Area and $C$ were chosen as the ecological metrics to rank the habitat patches based on their significance for connectivity. The metric Area identifies the significance of a habitat patch based purely on its area, without any reference to its inter-patch connectivity. Metric $C$ is a network scale metric which produces a scalar value representing the connectivity score of the whole network. The metric refers to the overall connectivity of the network as the sum of the connectivity between individual pairs of nodes (Mastisziw and Murray 2009). For this study the total connectivity of the network $(C)$ was calculated as

$$
C=\sum_{i}^{n} \sum_{j}^{n} a_{i} \times a_{j} \times p_{i j} \quad i=j .
$$

Here $C$ is the connectivity of the entire network, $a$ refers to the area of the individual patches $i$ and $j$, and $p_{i j}$ is the probability of dispersal between patches $i$ and $j$. The probability of dispersal and is defined as an exponential decay function of the effective distance between two patches (Hanski 1994, 1997; Urban and Keitt 2001; Pascual-Hortal and Saura 2006)

$$
p_{i j}=\exp \left(-k \cdot c w d i s t_{i j}\right)
$$

where $k$ is a constant and cwdist $\mathrm{ij}_{\mathrm{ij}}$ refers to the cost weighted distance along the least-cost path between patches $i$ and $j$. The value of the $k$ was estimated by using the assumption that the probability of dispersal is 0.05 when $c w d i s t$ equals its maximum value (Saura et al. 2011).

The node removal algorithm (see Introduction) was used to obtain an estimate of the contribution of each node to the total connectivity score of the network calculated by metric $C$. The contribution of the $i$ th node is indexed as delta $C(d C)$ and is given by:

$d C_{i}=\frac{C-C_{i}}{C} \times 100$ (Equation 4)

Here, $C$ is the connectivity value from the network scale metric with all nodes present and $C_{i}$ is the value with node $i$ removed. Thus nodes that make a larger contribution to the overall network connectivity will result in larger values of $d C_{i}$. 


\subsubsection{Integrated metrics}

The centrality metrics were applied to the weighted version for the fat-tailed dunnart habitat patch network. The links were weighted using the probability of dispersal $\left(p_{i j}\right)$ given in Equation 3 (Hanski 1994, 1997) and dispersal flux (flux $i j$ ) (Urban and Keitt 2001). Dispersal flux (henceforth referred to as simply flux) between a pair of habitat patches $i$ and $j$ is a function of their area and the probability of dispersal between them:

$$
\operatorname{flux}_{i j}=a_{i} \times a_{j} \times p_{i j} \quad \quad \text { (Equation 5) }
$$

Here $a_{i}$ and $a_{j}$ refer to the area of the habitat patches $i$ and $j$, and $p_{i j}$ refers to the probability of dispersal between them. The network that is weighted by probability of dispersal is termed $G_{p}$ and the network weighted by dispersal flux is termed $G_{\text {flux }}$.

The metrics $B C$ and $D C$ are then applied to the networks with the links weighted by the probability of dispersal $\left(B C_{p}, D C_{p}\right)$ and also with the links weighted by the dispersal flux $\left(B C_{\text {flux }}, D C_{\text {flux }}\right)$ using the thet package (Opsahl et al. 2010). In this case $\alpha$ was set to 0.5 to allow both the number of links attached to a given node and the weights of those links to count equally when estimating centrality metrics (Opsahl et al., 2010). Using these weighted networks allowed a more ecologically realistic estimation of a habitat patch's importance to dispersal.

Applying each of the eight metrics in turn allows the nodes in the network to be ranked according to the score assigned to them by each of the metrics. The most highly ranked nodes were then expected to be more important for the connectivity of a set of habitat patches, and so could be used to prioritize the maintenance of habitat connectivity and other conservation objectives. The correlation of the rankings for each of the nodes resulting from the application of the eight metrics was quantified using the Spearman's Rank Correlation Coefficient. The metric values assigned to the nodes were then able to be associated with each habitat patch for the fat-tailed dunnart. The geographical locations of highly ranked habitat patches (top 20 percent) were then compared to examine differences between the metrics. This enabled an examination into how the selected metrics differed in their predictions of the geographic location for important patches in the study area.

\section{Results}




\subsection{Construction of the habitat network for the fat-tailed dunnart}

An undirected network was developed for the fat-tailed dunnart in which nodes were linked to the adjacent nodes and the links were weighted by the effective distance (cwdist) of the corresponding least-cost path between them. The general characteristics of the resulting network are summarised in Table 1. The mean degree of the network nodes (the number of links directly attached to the given node) was 6.4 with a minimum node degree of one and a maximum of 41 .

Table 1 Characteristics of the fat-tailed dunnart habitat patch network

\begin{tabular}{lc} 
Network characteristics & 1309 \\
\hline Number of nodes & 4196 \\
Number of links & 6.4 \\
Mean degree of nodes & 41 \\
Maximum degree of node & 1 \\
Minimum degree of node & 878.7 \\
Mean Euclidean distance between the nodes (edge-to-edge) (metres) & 27220 \\
Maximum Euclidean distance between the nodes (metres) & 60 \\
Minimum Euclidean distance between the nodes (metres) & 13652 \\
Mean effective distance between the nodes (edge-to-edge) & 106452 \\
Maximum effective distance between the nodes & 120 \\
Minimum effective distance between the nodes & \\
\hline
\end{tabular}

\subsection{Measurement of the importance of species habitat patches}

When the probability of dispersal was used to weight the links, only 40 percent of the links had a weighted value greater than zero. The remaining links had a length greater than the estimated maximum dispersal ability of the dunnart (1000 meters) and their weight was set to zero meaning they were not processed by the software. In this process 186 nodes become entirely disconnected from the rest of the network and are termed 'isolated' nodes. The ratio of isolated nodes to the total is 0.14 .

Applying the eight metrics to the habitat patch network of the fat-tailed dunnart allowed the nodes for the network to be ranked by each metric. The correlation between each metric's rankings of the node scores is shown in Table 2. 
Table 2 Statistical comparison of the ranking of the habitat patches for the fattailed dunnart by applying the eight different metrics. $B C$ and $D C$ shows the application of metrics when the weights of the network is ignored. $B C_{p}$ and $D C_{p}$ shows the application of the metrics on the network weighted by probability of dispersal and $B C_{F l u x}$ and $D C_{F l u x}$ indicates the case that the network is weighted by flux. Correlations were calculated using the Spearman's rank correlation coefficient (rho).

\section{Ecological metrics Topological metrics Integrated metrics}

Area $\quad d C \quad B C \quad D C \quad B C_{p} \quad B C_{\text {Flux }} \quad D C_{p} \quad D C_{\text {Flux }}$

\begin{tabular}{|c|c|c|c|c|c|c|c|c|}
\hline \multirow{2}{*}{$\begin{array}{c}\text { Ecological } \\
\text { metrics }\end{array}$} & Area & 0.90 & 0.562 & 0.547 & 0.570 & 0.567 & 0.533 & 0.744 \\
\hline & $d C$ & & 0.571 & 0.659 & 0.565 & 0.520 & 0.668 & 0.917 \\
\hline \multirow{2}{*}{$\begin{array}{c}\text { Topological } \\
\text { metrics }\end{array}$} & $B C$ & & & 0.795 & 0.872 & 0.780 & 0.713 & 0.641 \\
\hline & $D C$ & & & & 0.730 & 0.610 & 0.943 & 0.807 \\
\hline \multirow{4}{*}{$\begin{array}{l}\text { Integrated } \\
\text { metrics }\end{array}$} & $B C_{p}$ & & & & & 0.80 & 0.681 & 0.618 \\
\hline & $B C_{\text {Flux }}$ & & & & & & 0.562 & 0.52 \\
\hline & $D C_{p}$ & & & & & & & 0.812 \\
\hline & $D C_{\text {Flux }}$ & & & & & & & \\
\hline
\end{tabular}

The correlation analysis revealed both differences and similarities in the way the habitat patches of the fat-tailed dunnart are ranked by metrics within the classes of the ecological, topological and integrated metrics (Table 2). Comparison of the correlation between classes revealed that ranking from the ecological metrics had a relatively low correlation with the ranking from the topology metrics. The strongest correlation between metrics of those two classes was between delta $C$ and $D C$, with a rho of 0.659 . The correlation of the habitat patch rankings based on the application of ecological class and integration class was still low; however, application of $D C_{F l u x}$ showed a relatively higher correlation with area of patches $(r h o=0.744)$. There was also a high correlation between the results from the application of delta $C$ and $D C_{F l u x}$ with the rho of 0.917 . This high correlation of results is due to the similarity between the method on which the node removal algorithm and degree centrality 
rank habitat patches combined with the fact that delta $C$ and $D C_{F l u x}$ both incorporate the area of habitat patches in their calculations.

In line with Baranyi et al. (2011), the results from this study show that ranking of nodes based on $B C$ either by weighting the links by probability of dispersal or flux were different from the ranking of nodes based on delta $C$ ( rho of $d C$ and $B C_{p}=0.565$, rho of $d C$ and $\left.B C_{F l u x}=0.520\right)$. When using $B C$, patch area was not included and the node ranks were based purely on the topology of the network and therefore the spatial distribution of the habitat patches played an important role. Even when comparing $B C_{F l u x}$ and $d C$, the correlation was relatively low even though both metrics incorporate the patch area in their calculations.

Comparing the topological and integrated metrics showed relatively high correlations (Table 2). Results from the application of $B C$ and $D C$ were highly correlated when the metrics were applied to the network weighted by probability of dispersal (for $B C$ and $B C_{p}$, rho $=0.872$; for $D C$ and $D C_{p}$, rho $=0.943$ ). This correlation becomes lower when the network is weighted by flux (for $B C$ and $B C_{F l u x}$, rho=0.780; for $D C$ and $D C_{F l u x}$, rho $=0.807$ ). This is due to the influence of the area of habitat patches used in the flux calculation, which is incorporated into $B C_{\text {Flux }}$ but not into $B C$. Thus compared to the probability of dispersal, the incorporation of the area of patches in flux calculation has a larger influence on the above mentioned correlations.

\subsection{Geographical location of the highly ranked habitats}

The eight metrics were applied to spatially rank the locations of the habitat patches of the fattailed dunnart, as this could be used for prioritizing areas for conservation activities. This prioritization is shown for the metrics $D C_{F l u x}$ and $B C_{p}$ and revealed the majority of the habitat patches with the greater contribution to habitat connectivity are geographically located at the margins of the study area (Figure 4). The urban fringe has greater coverage of native vegetation and larger habitat patches that potentially provide more suitable habitat for the species. By contrast, inside the Urban Growth Boundary the landscape is highly modified and more resistant to species movement, and so the habitat patches are contributing less to connectivity of the habitat patches. Figure 4 shows the patches ranked in the top 20 percent in dark green, which includes 262 out of the total 1309 patches. The application of $D C_{F l u x}$ showed farmlands in the north and in the south west near the bay as important to habitat connectivity (Figure 4, part a). Inside the Urban Growth Boundary, habitat patches near the 
northern edge are ranked in the top $20 \%$ by $D C_{F l u x}$. Applying $B C_{p}$ on the dunnart's network revealed that the farms and pastures in the north are also valuable in terms of their role as stepping stones or for movement of species (Figure 4b). Inside the Urban Growth Boundary the patches near the northern edge are recognised important stepping-stones for the species network. A number habitat patches ranked highly by $D C_{\text {Flux }}$ were assigned lower ranks when applying $B C_{p}$. Those habitat patches were located in north, northwest, west and near the edge of Urban Growth Boundary at the centre of study area.

Fig. 4 The ranking of habitat patches for the Fat-tailed Dunnart in relation to their geographical locations and the Urban Growth Boundary. The dark green patches have highest ranks as calculated by (a) $D C_{F l u x}(\mathrm{~b}) B C_{p}$. The ranking of nodes are rescaled to vary from 1 to 100 .

Using the $D C_{\text {Flux }}$ metric, the five top ranking patches cover 20 percent of the total area of species habitat within the study area. This is in contrast to using the $B C_{p}$, where for the same area the top 24, rather than just five, are incorporated. In fact the patches ranked in the top 20 percent, based on $D C_{F l u x}$ and $B C_{p}$ cover $88.6 \%$ and $75 \%$ of total area of species habitat respectively. This confirms the higher association of $D C_{F l u x}$ with area of habitats compared to the metric $B C_{p}$. Within the top 20 percent of patches, there was an overlap of 153 patches that were identified by both $D C_{F l u x}$ and $B C_{p}$. For the remainder of the top $20 \%$ there were 109 patches that differed between the two metrics (Table 3).

Table 3 The overlap in the number of patches ranked in the top $20 \%$ and bottom $80 \%$ based on the application of metrics $D C_{\text {Flux }}$ or $B C_{p}$

\begin{tabular}{|c|c|c|}
\hline & $\begin{array}{c}\text { Number of } \\
\text { common } \\
\text { patches } \\
\text { identified }\end{array}$ & $\begin{array}{l}\text { Number of } \\
\text { different patches } \\
\text { identified }\end{array}$ \\
\hline $20 \%$ & 153 & 109 \\
\hline $80 \%$ & 109 & 938 \\
\hline
\end{tabular}

\section{Discussion}


The application of the three classes of metrics to the network of habitat patches for the fattailed dunnart revealed that some of the metrics provide overlapping information whereas some others differ in the way they rank habitat patches. There is a high correlation between the results from delta $C$ and $D C_{F l u x}$ that suggests that potentially $D C_{F l u x}$ might be a useful surrogate for delta $C$ when ranking the nodes based on their contribution to connectivity. The metric $D C_{F l u x}$ identifies the patch significance based on its area, the probability of dispersal between adjacent patches and the number of weighted links attached to the patch. Thus $D C_{F l u x}$ aggregates multiple types of information within the one metric, making it a useful metric to apply to ranking the habitats within the heterogeneous urban landscapes.

In this study the metric $B C_{p}$ was shown to have a high correlation with the $B C$ applied to the unweighted network. $B C_{p}$ allows the assessment of patch importance based on topology of patches as well as probability of dispersal between patches, therefore is a suitable metric to be applied on the species network in heterogeneous urban landscape. However, compared to $B C_{p}, B C$ is easier to calculate. This is because $B C_{p}$ requires the calculation of the probability of dispersal based on the resistance of landscape parameters between patches which was achieved based on experts opinion and questionnaire survey in this study. Therefore in the case where the calculation of $B C_{p}$ is difficult in terms of data provision, $B C$ can be used as a surrogate metric in place of $B C_{p}$, and at least for the fat-tailed dunnart in our study area, the ranking of patches prioritized in the connectivity network will be similar. The results from $D C$ and $D C_{p}$ were highly correlated suggesting there is no need for the calculation of effective distance as a function of landscape resistance and species dispersal abilities and the application of $D C$ is sufficient and needs lower data requirements. The application of $B C$ and $B C_{p}$ has advantageous as the metric enables identifying the important stepping-stones within the urban growth boundary where the habitat patches quite small in size.

In the case where the conservation strategy is to maintain large stepping-stones or large habitat patches, then $B C_{F l u x}$ and $D C_{F l u x}$ is applicable. These are the cases where the structure of urban landscape is heterogeneous in terms of size of habitat patches and so metrics that incorporate the area of the patches should be considered. For cases where the size of habitat patches does not have a considerable variation, then application of purely topological metrics such as $D C$ and $B C$ is sufficient and ecologically informative $\left(B C\right.$ and $B C_{F l u x}, r h o=0.780$; for $D C$ and $D C_{\text {Flux }}$, rho $\left.=0.807\right)$. 
Results from the application of metric $B C_{F l u x}$ showed very low association with results from the application of $D C_{F l u x}$ and thus both metrics show differing information on the significance of habitat patches. The difference between the way $B C$ and $D C$ rank the patches was shown in previous studies that revealed that $B C$ identifies important stepping stones whereas $D C$ detects important habitat patches (Estrada and Bodin 2008; Baranyi et al. 2011). In this study by even incorporating the area and probability of dispersal we still noticed that the resultant prioritization based on the two metrics are different and so may apply for different study purposes.

The metric $C$ and flux that were used to measure the network connectivity and to weight the links in calculation of degree centrality, placed more emphasis on the area of habitats compared to the probability of dispersal between the habitats. This fact was also highlighted by Laita et al. (2011). There is still uncertainty in relative influence of the area of an individual habitat patch with respect to the probability of dispersal within the flux. By reducing the influence of the patch area and the emphasis on probability of dispersal, the influence of effective distances calculated by least-cost modelling will be increased. This influence can also be different per species and species-specific movement characteristics. The justification of the relative influence of area and probability of dispersal within the flux metric highlights an area for further investigation for future studies specially those that connectivity is assessed for multiple species.

\section{Conclusions}

This study compared multiple metrics for identifying habitat patches which are important in maintaining connectivity of a species habitat patch network. We applied both ecological and topological metrics to the habitat networks of the fat-tailed dunnart within Greater Metropolitan Melbourne to estimate the contribution of individual habitat patches to the connectivity of the network. This study also defined a new class of metrics that integrate both the topological and ecological attributes of habitat patches by incorporating the effective distance rather than just the Euclidean distance into the dispersal kernel metric. Statistical comparison of patch ranking based on each of the metrics revealed similarity and differences between the ways each metric rank patches. This study revealed that some metrics such as $d C$ and $D C_{F l u x}$ results in similar rankings of patch importance and they can use interchangeably. 
However, other metrics such as $D C_{F l u x}$ and $B C_{F l u x}$ differ in the way they rank patch importance and provide complementary information. Use of $D C_{p}$ and $B C_{p}$ resulted in prioritization similar to topological metrics, $D C$ and $B C$. Therefore this study suggested integrated metrics when incorporation of the patch area is necessary in analysis otherwise the well applied topological metrics which need lower data requirement are sufficient and informative. Overall this study illustrated the importance of understanding the application of metrics to suit the research objectives before method and metrics being selected.

\section{Acknowledgements}

The Department of Sustainability and Environment of the Victorian Government supplied the input data for this study. Special thanks to Dr. Matt White who prepared the input habitat maps for this study. Thanks go to Dr. Peter Menkhorst who provided consultation on the spatial ecology of the fattailed dunnart and filled in the questionnaires. Special thanks to Dr. Brad McRae and Dr. Bronwyn Rayfield for their valuable guidance on the use of LinkageMapper and least cost modelling. Sincere thanks go to Mr. Hossein Pourali and Mr. Stephen Page for their technical advice on ArcGIS. Dr. Tore Opsahl gave valuable guidance on the use of tnet package of software R. Ascelin Gordon was supported by the Australian Research Council Centre of Excellence for Environmental Decisions. The Iranian Ministry of Science, Research and Technology sponsored this research.

\section{References}

Adriaensen F, Chardon JP, De Blust G, Swinnen E, Villalba S, Gulink H, Matthysen E (2003) The application of 'least-cost' modelling as a functional landscape model. Landscape and Urban Planning 64:233-247

Baranyi G, Saura S, Podani J, Jordán F (2011) Contribution of habitat patches to network connectivity: Redundancy and uniqueness of topological indices. Ecological Indicators 11 (5):1301-1310

Beier P, Majka DR, Spencer WD (2008) Forks in the road: Choices in procedures for designing wildland linkages. Conservation Biology 22 (4):836-851

Bennett A (2003) Linkages in the landscape: the role of corridors and connectivity in wildlife conservation. The IUCN forest conservation programme; conserving forest ecosystems, vol 1. IUCN, Gland, Switzerland and Cambridge, UK,

Bodin O, Norberg J (2007) A network approach for analyzing spatially structured populations in fragmented landscapes. Landscape Ecology 22:31-44 
Bodin O, Saura S (2010) Ranking individual habitat patches as connectivity providers: Integrating network analysis and patch removal experiments. Ecological Modelling 221:2393-2405

Cantwell MD, Forman RTT (1993) Landscape graphs: Ecological modelling with graph theory to detect configurations common to diverse landscapes. Landscape Ecology 8 (4):239-255

Carroll C, McRae BH, Brookes A (2012) Use of linkage mapping and centrality analysis across habitat gradients to conserve connectivity of Gray Wolf population in Western North America. Conservation Biology 26 (1):78-87

Collinge SK, Forman RTT (1998) A conceptual model of land conversion processes:

Predictions and evidence from a microlandscape experiment with grassland insects. Oikos 82 (1):66-84

Dale MRT, Fortin MJ (2010) From Graph to Spatial Graph. The Annual Review of Ecology, Evolution, and Systematics 41:21-38

Department of Planning and Community Development (2002) Melbourne 2030: Planning for sustainable growth. Victorian Government Department of Planning and Community Development, Melbourne

Department of Sustainability and Environment (2007) Advisory list of threatened vertebrate fauna in Victoria. Melbourne

Department of Sustainability and Environment (2009a) Advisory list of threatened invertebrate fauna in Victoria. Melbourne

Department of Sustainability and Environment (2009b) Delivering Melbourne's Newest Sustainable Communities; Strategic Impact Assessment Report. Melbourne

Estrada E, Bodin O (2008) Using network centrality measures to manage landscape connectivity. Ecological Applications 18 (7):1810-1825

Farina A (2000) Landscape Ecology in Action. Kluwer Academic Publishers, Dordrecht

Ferrari JR, Lookingbill TR, Neel MC (2007) Two measures of landscape-graph connectivity: assessment across gradients in area and configuration. Landscape Ecology 22:13151323

Foltête JC, Clauzel C, Vuidel G, Tournant P (2012) Integrating graph-based connectivity metrics into species distribution models. Landscape Ecology 27 (4):557-569

Freeman LC (1978) Centrality in social networks conceptual clarification. Social networks 1 (3):215-239. doi:10.1016/0378-8733(78)90021-7

Frey H (1991) Energetic significance of torpor and other energyconserving mechanisms in free-living Sminthopsis crassicaudata (Marsupialia: Dasyuridae). Australian Journal of Zoology 39:689-708 
Galpern P, Manseau M, Fall A (2011) Patch-based graphs of landscape connectivity: A guide to construction, analysis and application for conservation. Biological Conservation 144 (1):44-55

García-Feced C, Saura S, Elena-Rosselló R (2011) Improving landscape connectivity in forest districts: A two-stage process for prioritizing agricultural patches for reforestation. Frontiers in Ecology and Management 261:154-161

Gordon A, Simondson D, White M, Moilanen A, Bekessy SA (2009) Integrating conservation planning and landuse planning in urban landscapes. Landscape and Urban Planning 91:183-194

Hahs AK, McDonnell MJ (2006) Selecting independent measures to quantify Melbourne's urban-rural gradient. Landscape and Urban Planning 78:435-448

Hall LS, Krausman PR, Morrison ML (1997) The habitat concept and a plea for standard terminology. Wildlife Society Bulletin 25 (1):173-182

Hamer AJ, McDonnell MJ (2010) The response of herpetofauna to urbanization: Inferring patterns of persistence from wildlife databases. Austral Ecology 35 (5):568-580

Hanski I (1994) A practical model of metapopulation dynamics. Journal of Animal Ecology 63:151-162

Hanski I (1997) Predictive and practical metapopulation models: the incidence function approach. In: Tilman D, Kareiva P (eds) Spatial ecology. Princeton University Press, Princeton, USA, pp 21-45

Harary F (1969) Graph Theory. Addison-Wesley Reading Mass,

Henderson MT, Merriam G, wegner J (1985) Patchy Environments and Species Survival: Chipmunks in an Agricultural Mosaic. Biological Conservation 31:95-105

Keitt TH, Urban DL, Milne BT (1997) Detecting critical scales in fragmented landscapes. Conservation Ecology 1 (1):4

Kindlmann P, Burel F (2008) Connectivity measures: a review. Landscape Ecology 23:879890

Laita A, Kotiaho JS, Mönkkönen M (2011) Graph-theoretic connectivity measures: What do they tell us about connectivity? Landscape Ecology 26 (7):951-967

Lindenmayer D, Hobbs RJ (eds) (2007) Managing and Designing Landscapes for Conservation: Moving from Perspectives to Principles (Conservation Science and Practice) Blackwell Publishing,

Magle SB, Theobald DM, Crooks KR (2009) A comparison of metrics predicting landscape connectivity for a highly interactive species along an urban gradient in Colorado, USA. Landscape Ecology 24 (2):267-280

Mastisziw TC, Murray AT (2009) Connectivity change in habitat networks. Landscape Ecology 24:89-100 
McIntyre S, Hobbs R (1999) A framework for conceptualizing human effects on landscapes and its relevance to management and research models. Conservation Biology 13 (6):1282-1292

McRae BH, Kavanagh DM (2011) Linkage Mapper Connectivity Analysis Software. The Nature Conservancy. http://www.circuitscape.org/linkagemappe.

Menkhorst PW, Bennett A (eds) (1995) Mammals of Victoria: distribution, ecology and conservation. Oxford University Press, Melbourne

Menkhorst PW, Kenight F (2001) A Field Guide to the Mammals of Australia. Oxford University Press, Australia

Morton S (1976) Ecological and Physiological Studies of Sminthopsis Crassicandata. Melbourne University, Melbourne

Noss RF, Dobson AP, Baldwin R, Beier P, Davis CR, Dellasala DA, Francis J, Locke H, Nowak K, Lopez R, Reining C, Trombulak SC, Tabor G (2012) Bolder Thinking for Conservation. Conservation Biology 26 (1):1-4

O'Brien D, Manseau M, Fall A, Fortin MJ (2006) Testing the importance of spatial configuration of winter habitat for woodland caribou: An application of graph theory. Biological Conservation 130 (1):70-83

Opsahl T, Agneessens F, Skvoretz J (2010) Node centrality in weighted networks: Generalizing degree and shortest paths. Social Networks 32 (3):245-251

Pascual-Hortal L, Saura S (2006) Comparison and development of new graph-based landscape connectivity indices: towards the priorization of habitat patches and corridors for conservation. Landscape Ecology 21:959-967

Rayfield B, Fortin M-J, Fall A (2011) Connectivity for conservation: a framework to classify network measures. Ecology 92 (4):847-858

Read DG (1984) Movements and home ranges of three sympatric dasyurids, Sminthopsis crassicaudata, Planigale gilesi and P. tenuirostris (Marsupialia), in semiarid western New South Wales. Australian Wildlife Research 11 (2):223-234

Rothley KD, Rae C (2005) Working backwards to move forwards: Graph-based connectivity metrics for reserve network selection. Environmental Modelling and Assessment 10:107-113

Saura S, Estreguil C, Mouton C, Rodríguez-Freire M (2011) Network analysis to assess landscape connectivity trends: Application to European forests (1990-2000). Ecological Indicators 11:407-416

Saura S, Pascual-Hortal L (2007) A new habitat availability index to integrate connectivity in landscape conservation planning: Comparison with existing indices and application of case study. Landscape and Urban Planning 83:91-103

The Economist Intelligence Unit (2011) A Summary of the Liveability Ranking and Overview. www.eiu.com. www.eiu.com. Accessed 3rd November 
Urban D, Keitt T (2001) Landscape connectivity: a graph-theoritic perspective. Ecology 82 (5):1205-1218

Urban DL, Minor ES, Treml EA, Schick RS (2009) Graph model of habitat mosaics. Ecology Letters 12:260-273

Vasas V, Magura T, Jordan F, Tothmeresz B (2009) Graph theory in action: evaluating planned highway tracks based on connectivity measures. Landscape Ecology 24:581586

Victorian Environmental Assessment Council (2010) Remnant Native Vegetation Investigation; Discussion Paper.

Wheater PC (1999) Urban Habitats. Routledge, London 